Die Veroffentlichungen ther die Vogelwelt Pommerns.

Wernich 1885.

Weste 1894, 1895, 1896. Wibelitz 1907.

Wiese 1855, 1857, 1859, 1860, 1861, 1868, 1867, 1875, 1876, 1885, 1886, 1887.

v. Wilamowitz 1914.

Wilhelm 1888.
Wilke 1888, 1889, 1890,

1898, 1895, 1898, 1899, $1908,1911$.

Winkelmann 1908, 1910, 1911, $1912,1918$.

Witzmann 1895.

Wolff 1918, 1914.

Wrede 1828.

Wüstnei 1900, 1901.
Z.

Zander 1915.

Zielinsky 1896, 1908.

Ziemer 1884, 1885, 1887, $1888,1898,1894$.

Zimmermann 1895.

Zuchors 1907.

\title{
Die Rohrsănger des Leipziger Flachlandsgebietes mit besonderer Beriicksichtigung ihres Vorkommens in den Flufsgebieten der weifsen Elster, Pleifse, vereinigten und Zwickauer Mulde nach dem sächsischen Berglande und Erzgebirge zu.
}

\section{Von Rich. Schlegel.}

Ich erinnere mich heute, nach ca. 25 Jahren, noch recht lebhaft und besonders gern der Freude, die sich meiner bemächtigte, als ich nach meiner Versetzung aus dem Erzgebirge, resp. sächsischen Berglande nach Leipzig im hiesigen Niederungsgebiete zum ersten Male mit dem kleinen Rohrsängervolk die ersten Bekanntschaften in der Natur anzuknüpfen ausgiebig Gelegenheit fand. Wenn auch in den bis dahin beobachteten Gebieten, besonders im mittelsächsischen Berglande, am Fufse des Erzgebirges, gröfsere und kleinere stehende und fliefsende Gewässer keineswegs selten sind, auch das Gebiet der Zwickauer Mulde stellenweise besonders gern in mein Beobachtungsbereich gezogen wurde, so war es mir bis dahin beim Fehlen alles Rohrschilfes und aller mit Weiden und anderem Sumpfgewächs bestandener Ausschachtungs- und Niederungsgebiete nie geglückt, das Vorkommen irgend einer Rohrsängerart jemals feststellen zu können. Denn alle Rohrsängerarten, wenn man vielleicht vom Sumpfrohrsänger absehen will, der an seine Wohngebiete ja längst nicht mehr die speziellen ursprünglichen Anforderungen stellt wie ehedem, sondern sich veränderten Verhältnissen anzupassen verstand, liefern ein treffendes Beispiel dafür, wie ganz bestimmte Arten auch an ganz bestimmte Vegetationsformationen, wenn man auch kleine, charakteristisch bewachsene Flächen einmal so nennen darf, gebunden sind, mehr abhängig von diesen als von einer vertikalen Gliederung, vom Bodenrelief selbst. Bei meiner Studie lag mir besonders daran, einmal die Besiedelungsdichte innerhalb der drei genannten Flufsgebiete kennenzulernen, andernteils aber auch das Vordringen der einzelnen Arten gebirgswärts zu verfolgen und die südlichsten Brutgebiete innerhalb dieses Gebietes festzulegen. Wenn Faunisten, denen bei der zu berücksichtigenden Literatur auch eingehende 
persönliche Erfahrungen zur Seite stehen müfsten, sich ähnliche Untersuchungen für andere Arten sächsischer Vögel unterziehen würden, erhielten wir damit interessante ornithologische Grenzlinien für die einzelnen Arten und Nachweise darüber, wie das Bodenrelief und die damit in Beziehung stehenden veränderten Vegetationsverhältnisse den einzelnen Arten einen Grenzwall entgegenzusetzen vermögen. Wenden wir uns nun im speziellen den Verhältnissen hinsichtlich der Rohrsänger zu, so ergibt sich bei Berücksichtigung der einschlägigen Literatur nach meinen Feststellungen betreffs der einzelnen Arten folgendes Bild.

\section{Acrocephalus palustris (Bechst.).}

Der Sumpfrohrsänger ist im Leipziger Gebiete an geeigneten Örtlichkeiten ziemlich häufig anzutreffen. Ziemlich zahlreich traf ich ihn im Gebiete bei Möckern-Wahren-Lützschena zwischen Luppe und Elster. Die dortigen, mit allerlei Strauchgewirr, Weiden, Nesseln, Sumpf- und Niederungspflanzen bestandenen Ausschachtungen und Ufer gewähren ihm alle erforderlichen Existenzbedingungen. Offenbar darf er vielleicht in derselben Häufigkeit auch in den güustigen Elsterniederungen bei Knauthain Eythra, südlich von obigem Gebiete auftreten, was mir R. Müller mündlich versichert. Häufig setzt der Vogel wieder ein im Pleifsenund Göselbachgebiet bei Markkleeberg, Crostewitz, Gaschwitz, Deuben, Zehmen. Bei letztgenannten Orten traf ich ihn auch als Bewohner der Getreidefelder an. Eine Wanderung an den Ufern der Pleifse innerhalb dieser Örtlichkeiten ist besonders an Morgenund Abendstunden bei dem besonders um diese Zeit immer fleifsigen Sänger für Gesangskundige recht genufsreich und lohnend. Auch im Gebiete der Parthe bei Mockau und Schönefeld traf ich diesen niedlichen Sänger regelmäfsig an. Von hier aus besiedelt er ebenfalls die umliegenden Getreidebaudistrikte. Wie anderwärts, bewohnt unser Vogel auch die östlichen und südöstlichen Ackerbaugebiete des Leipziger Bezirkes. Als regelmäfsige Erscheinung begegnet man ihm somit auf jeder Exkursion zwischen Dölitz und Probstheida, bei Zweinaundorf und Paunsdorf, bei Holzhausen und östlich davon im Gebiete des Zauchgrabens. Im Gundorfer Ausschachtungs- und Luppengebiet, wo den Sumpfrobrsänger Dr. Hesse in wechselnder Häufigkeit beobachtete (Ornith. Monatsber. 1904/05, J. f. 0. 1907-09), traf ich den Vogel ebenfalls, wenn auch mehr zerstreut, an. Ohne irgendwelchen Grund für die Tatsache zu finden, tritt der Vogel, was besonders auch für die Sperbergrasmücke gilt, nicht alljährlich in gleicher Häufigkeit auf, was auch Dr. Hesse und Höpfner für Grimma hervorheben. So fand ich, um nur einen Fall aus meinen diesbezüglichen Aufzeichnungen herauszugreifen, in einem meiner Kuckucksbeobachtungsgebiete 1905 nur 1 Pärchen an gegen 4 im Jahre vorher. 1905 trat der Vogel auch seltener im Möckernschen Gebiete auf. 
Dr. Hesse, der den Sumpfrohrsänger, etwas im Widerspruche zu meiner Ansicht, für das Leipziger Gebiet einen unregelmäIsigen, selteneren Brutvogel nennt, stellt sein Vorkommen ferner fest und zwar - vermutlich noch während des Zuges teilweise auch im Unterholze inmitten des Waldes, sogar im Fichtengebüsch im Rosental, bei Schkeuditz, Mafslau, Lindental, Gohlis, Lützschena, in der Nähe des Bienitzhügels, in den Getreidefeldern bei Rückmarsdorf und Lindental, bei Connewitz - Lösnig, an der Parthe bei Cradefeld, bei Kleinpomfsen, Grethen, Belgershain, Gaschwitz, Crostewitz und Markklenberg. Nach Professor A. Voigt (Anl. z. Stud. d. Vogelstimmen, Beil. z. Jahresber. der 1. städt. Realsch. z. Leipzig, Ostern 1892) finden wir den Vogel viel um Leipzig an den Ausstichsümpfen bei Grofszschocher, zwischen Möckern und der Elster u. a. O. Fr. Lindner (Ornith. Monatsschr. 1884 und 1886) fand den Sänger bei Möckern in 6-8 Paaren, und scheint er nach seiner Meinung das ganze Elstertal von Gera bis Leipzig zu bewohnen. Diese Tatsache registriert schon Prof. Liebe (J. f. 0. 1878), der den Vogel in Auweidicht unterhalb Gera, im unteren und mittleren Elstertale feststellt, ihn aber noch als seltenen Brutvogel im Felde - sein Auftreten dort nur vielleicht als Gast - bezeichnet. Im Rohrbacher Teichgebiet stellt ihn Prof. Hennicke (Ornith. Monatsschr. 1891) in grofser Individuenzahl fest und vermutet als Sommervogel sein Brüten. In einer späteren Arbeit (Ornith. Jahrb. 1894) aber nennt er ihn den am wenigsten häufigen unter den Rohrsängern, doch nicht selten. Auch Dr. Hesse stellt sein Vorkommen dort fest. Fritzsche (Jahresberichte f. Sachsen) berichtet über ein Nest Ende der 60 er Jahre bei Pegau an der Elster im Weidengebüsch. Im Haselbach-Breitinger Teichgebiet beobachtete ihn Dr. Proft nach mündlicher Mitteilung durchschnittlich in ca. 3 Pärchen. Die umliegenden Getreidefelder beherbergen den Vogel ebenfalls. Dr. Koepert (J. f. O. 1896) verzeichnet das Brüten des Sumpfrohrsängers im Altenburger Gebiet als immerhin seltener. Er wurde nach Heller 1887 bei Göfsnitz am Pleifsenufer, bei Merlach und von Dr. Koepert selbst 1895 an den Wilchwitzer Teichen festgestellt. Für das Frohburger Teichgebiet stellt das Vorkommen des Sumpfrohrsängers u. a. auch Dr. Bräls fest (B. IV. der Mitt. des Landesvereins Sächs. Heimatssch.). Seltener ist das Auftreten des Vogels im Muldengebiet. Hülsmann (Ornith. Monatsschr. 1889 und sächs. Berichte) verzeichnet ihn nicht für das Gebiet bei Wurzen, aber nach Heym (Berichte für Sachsen) ist er "Sommerbrutvogel" dort. Jedenfalls ist die Annahme nicht unbegründet, dafs eine Verwechslung mit dem Teichrohrsänger vorliegt. Prof. Thienemann aber teilte mir am 10. VI. $1896 \mathrm{mit}$, dals er den Vogel bei Altenbach-Wurzen festgestellt habe. In der Gegend von Grimma wird er von Prof. Voigt und Höpfner (Berichte f. Sachsen) für Altenhain und von Höpfner für Seehrhausen festgestellt. Wollen wir nach das Wermsdorfer Teichgebiet 
östlich der Vereinigten Mulde in Betracht ziehen, so ist er nach Heyder (Ornith. Monatsschr. 1909) vereinzelt im Schilf und hänfiger im Feld anzutreffen. Alle weiteren Beobachter im Muldengebiet schweigen nach den Sächs. Berichten über das Vorkommen dieses Vogels. Nur Berge (Die Vögel der Umg. von Zwickau), dessen Beobachtungsgebiet sich nordwärts bis Crimmitschau, Glauchau, Hohenstein erstreckt, berichtet, dafs er ein seltener Brutvogel sei, den er im Muldenröhricht bei Wermsdorf feststellte. Während meiner Beobachtungszeit in der Gegend von Hohenstein - Ernsttal in den Jahren 1888-91 konnte ich den Sumpfrohrsänger nirgends auffinden, und auch die späteren Jahre, während welcher ich im Gebiete besuchsweise öfters und auch längere Zeit beobachtete, habe ich den Vogel niemals angetroffen. Umso freudiger überrascht war ich, als ich im Sommer 1915 während des Sommerurlaubes 5 singende Männchen im Gebiete des Lungwitzbaches und eines Zuflusses, des Goldbaches, verhören konnte. Die Vögel sangen alle in dort ausnahmsweise hochhalmigen Haferfeldern. Da ein Ausbreiten vom vereinigten Muldengebiet nach südwärts infolge der für den Rohrsänger weniger günstigen Bodenverhältnisse kaum anzunehmen ist, komme ich zur Überzeugung, dafs ein östliches, bezw. südöstliches Vorrücken, vom Elster- und Pleifsengebiete aus, stattgefunden hat. Wünschen wir dem kleinen Wanderer ferneres Glück zum Weiterkommen! Ziemlich spärlich liegen Nachrichten über die Zugverhältnisse vor. Ich selbst habe hierüber keinerlei Aufzeichnungen gemacht. Prof. Voigt gibt die Ankunft für Mitte Mai an. Wie zu erwarten ist, lärst uns diesbezüglich auch der gründliche Dr. Hesse nicht im Stich, indem er die Zeit seines Aufenthaltes von Mitte Mai bis Mitte September registriert. Umsoreichlicher sind meine Brutnotizen. Im Aubezirke fand ich zahlreiche Nester hauptsächlich in Nesseln, Weiden-, Berberitzenund je einmal auch in Herbstastern- und Schwarzdornschöfslingen immer niedrig über dem Boden. Ende Mai bis Mitte Juni dürfte die normale Legezeit sein. Unter besonders günstigen Umständen finden wir die Gelege schon im letzten Drittel des Mai vollständig vor. Eierfunde notierte ich für:

29. V. fertiges Nest.

31. V. 1 frisches Ei.

1. VI. 5 Eier ca. 6 Tage bebr.

2. VI. fertiges Nest.

5. VI. 5 Eier, schwach bebr.

6. VI. 3 Eier, frisch.

7. VI. 5 Eier, frisch.

8. VI. 5 Eier, schwach bebr.

10. VI. 5, 5 Eier, schwach bebr.

4, 5 Eier, stärker bebr.

11. VI. 5 Eier, ca. 4 Tage bebr.
12. VI. 5 Eier, stark bebr. (Thienemann).

14. VI. 4, 3, 3, 3, 1, 1 frische Eier.

22. VI. 5, 4-5 Tage alte Junge, 4 Eier, stark bebr.

23. VI. 5 Junge mit Kielen.

28. VI. 5 Eier, stark bebr., $3 \mathrm{fr}$. Eier, 1 Ei mit Cuculusei.

30. VI. 3 Eier, zieml. stark bebr. 7. VII. 5 ca. 5 Tage alte Junge. 19. VII. 3 frische Eier.

Die normale Eierzahl im Gelege beträgt $4-5$, häufiger 5 als 4. Sechsergelege, wie ich eins aus Dänemark besitze, sind 
mir für hier und auch im Bernburger Gebiet, wo ich Gelege mit A. Bähr recht häufig fand, nicht bekannt geworden. In 3 Fällen fand ich bei Möckern-Wahren im Neste des Sumpfrohrsängers auch Kuckuckseier. R. Müller fand sie bei demselben Pfleger bei Markkleeberg-Crostewitz, und Dr. Proft teilt mir mit, dafs der Sumpfrohrsänger auch im Breitingen-Haselbacher Teichgebiet ausnahmsweise als Kuckucksbrutpfleger fungiert.

\section{Acrocephalus strepera strepera (Vieill.).}

Der Teichrohrsänger, in seinem Auftreten lediglich aus Rohr gebunden, kommt im Gebiete allenthalben dort zahlreich vor, wo Teiche, Flufsläufe, Ausschachtungen und Tümpel mit Rohr und Weidicht bewachsen sind. In nächster Nähe ist das Tier häufig nach Dr. Hesse ebenfalls häufiger Brutvogel -, zuweilen recht bäufig im Möckernschen Ausschachtungsgebiet, z. B. in den Jahren 1893-95, für welche Jahre ganz eingehende diesbezügliche Aufzeichnungen vorliegen. Regelmälsig ist der Vogel auch in den Gundorfer Lachen anzutreffen. Infolge Zurücktretens des Rohres an den Ufern der Pleifse im Südgebiete finden wir hier den Vogel auch weniger zahlreich. Sobald aber kleine Rohrbestände einsetzen, finden wir dort den Rohrsänger auch als charakteristische Erscheinung wieder, so beispielsweise am Markkleeberger Wehre und an der Stelle, wo der Göselbach bei Markkleeberg sich stark der Pleifse nähert. Ferner beobachtete ich diesen Rohrsänger in den Ausschachtungen bei Wabren und Grofszschocher, ziemlich häufig dann wieder im Parthegebiet bei Schönefeld-Mockau und meist recht häufig in den Teichgebieten von Machern, Lübschütz, Breitingen und Rohrbach. 1904 registriert ihn Dr. Hesse für Gundorf als selten, 1905 aber traten nach ihm dort ca. 8 Brutpaare auf. Beobachtet wurde von ihm unser Vogel auch an verschiedenen Stellen im Unterholze des Waldes in der Nähe des Wassers. Prof. Voigt erwähnt nur, dafs er fast ausschliefslich Gewässer bewohnt, mit recht viel und dicht stehendem Schilfrohre. Jacobi und Lohse (Sächs. Ber.) stellen ihn als Brutvogel bei Connewitz und Schleufsig fest. Nach R. Müller (mündl. Mitt.) ist er auch häufig bei Knauthain und Eythra. Fr. Lindner verzeiehnet ihn für Möckern, und Fritzsche sagt, dafs er bei Pegau Rohrspatz genannt werde. Für Frohburg bezeichnet ihn Sperling (Sächs. Ber.) als häufig, und auch von Müller und Ulbricht (Sächs. Ber.) wird er für Borna als dort brütend angefülırt. Prof. Hennicke führt ihn für das Rohrbacher Teichgebiet als „in grofser Individuenzahl" und ,in ungeheurer Menge" als vorkommend an. 1905 stellte Dr. Hesse dort 35 Brutpaare fest, sagt aber für 1908, dafs der Bestand an Drossel- und Teichrohrsängern die letzten Jahre bedeutend abgenommen habe. Prof. Liebe weist darauf hin, dafs er sein Gebiet bis zum Frankenwalde ausdehne und nennt ihn den gemeinsten Rohrsänger im Gebiet. Er verzeichnet ihn als 
brütend bei Gera, Greiz und Zeitz, für letzteren Ort auch als im rohrfreien Weidicht brütend. Dr. Koepert bezeichnet den Teichrohrsänger als häufigen Brutvogel an den Wilchwitzer, Haselbacher, Oberlödaer und Hainspitzer Teichen. Im Gebiete der Vereinigten Mulde wird er von Höpfner für Grimma, Nimbschen und Altenhain festgestellt, und auch Hülsmann bezeichnet ihn für Wurzen als "Sommerbrutvogel". Nach Heyder tritt er vereinzelt an allen Hubertusburger Teichen auf, doch nirgends häufig. Auch im Zwickauer Gebiet kommt er nach Berge als Brutvogel vor an Gewässern mit dichtem Rohr und Gesträuch. Etwas zahlreicher liegen für den Teichrohrsänger die Zugnotizen vor. Päfsner berichtet als Ankunftszeiten „Anfangs Mai“" und 5. V. Höpfner notierte den ersten Gesang am 2. V. Roux will nach mir gewordenen mündlichen Mitteilungen 1895 seine Ankunft schon am 23. IV. festgestellt haben. Als Aufenthaltszeit notierte Dr. Hesse für verschiedene Jahre 8. V.-23. VIII., 12. V.--31. VIII., 12. V.-15. IX., 11. V.-1. X. Als Abzugsdatum gibt Päfsler einmal den 1. IX. und Höpfner den 24. IX. an. Jacobi und Lohse sagen, dafs er uns in den letzten Tagen des August wieder verläfst. Die von mir zahlreich aufgefundenen Nester standen ausnahmslos alle im Rohrschilf und sind aufser nach dem Standort sofort auch nach dem Baumaterial sicher zu bestimmen, da zum Bau immer nur vertrocknete Rispen des Rohrschilfes Verwendung finden. Durchschnittlich stehen die Nester immer höher als die seines Vetters palustris. Hinsichtlich der Eierzahl im Gelege ist das Verhältnis umgekehrt wie bei palustris. Viel häufiger sind die Gelege mit 4 Eiern vollzählig als mit 5, obwohl 5 Eier im Gelege nicht als Seltenheit zu bezeichnen sind. Über die Brutzeit selbst geben nachstehend verzeichnete Daten das beste Bild.

22. V. fertiges Nest.

28. V. 4 fr. Eier, vollendetes Nest, beg. Nest, fertiges Nest, 3 fr. Eier, 2 fr. Eier mit Kuckucksei.

31. V. 4 Eier, wenig bebr.

2. VI. 4 Eier, zur Hälfte bebr., $1 \mathrm{fr}$. Ei, $2 \mathrm{fr}$. Eier in demselben Neste, dem am 28. V. das Gelege mit Kuckucksei entnommen wurde.

10. VI. 4 fr. Eier, 2 fr. Eier, 5 stärker bebr. Eier, 5 schw. bebr. Eier.

12. VI. 4 stark bebr. Eier.

13. VI. 2 bebr. und 2 faule Eier mit Kuckucksei, 3 zieml. stark bebr. Eier.

14. VI. 4 fr. Eier, 4 fr. Eier.

7. VII. 2 fr. Eier, 2 Nester mit je 3,5-6 T. alten J., 1 neues Nest.

19. VII. Ausgefl. Junge, 2 Nester mit halberw. J.

31. VIII. Ausgefl. Junge werden noch gefüttert (Dr. Hesse).

Aus den Aufzeichnungen ist ersichtlich, dafs die Brutzeit im letzten Drittel des Mai einsetzt und normalerweise in der Zeit 
bis Mitte Juni kulminiert. Die bis in den Juli hinein sich erstreckende Brutpflege hat ihren Grund in nachgelegten Eiern erstmalig gestörter Brutpaare; denn, ungestört, schreitet auch der Teichrohrsänger nur einmal im Jahre zur Brut. Nicht selten ist dieser Rohrsänger im Leipziger Gebiet der Brutpfleger vom Kuckuck, an den Lübschützer, Breitingen-Haselbacher Teichen sogar in erster Linie.

\section{Acrocephalus arundinaceus arundinaceus (L.).}

Der Drosselrohrsänger ist im Leipziger Gebiete eine allbekannte und im allgemeinen häufigere Erscheinung. So traf ich das Tier in einzelnen Pärchen oder ziemlich häufig bei Möckern und Wahren an, ferner in einem kleinen Rohrbestande bei Dösen und regelmälsig auch an der Pleifse bei Markkleeberg und Crostewitz. Hier beobachtete ich auch, wie die Rohrdrossel gern die Gebüsche der Wiesen in der Nähe des Wassers besucht, wie Dr. Hesse den Vogel, in Baumkronen singend, wiederholt beobachtete. Als häufige Erscheinung notierte ich die Rohrdrossel für Machern, Lübschütz sowie für die Breitingen-Haselbacher Teiche, an letzten Orten zeitweise recht häufig. Prof. Voigt berichtet uber das Vorkommen bei Grofszschocher und Möckern, Barneck, desgleichen Fr. Lindner für Möckern. Dr. Hesse, der die Rohrdrossel einen häufigen Brutvogel nennt, stellte 1905 in Gundorf, ca. 10 Brutpaare fest. Wenn man der Notiz Fritzsches (Sächs. Ber.) die nötige Bedeutung beimessen will, müfste die Rohrdrossel vor Jahrzehnten im Leipziger Gebiete noch selten vorgekommen sein; denn er erwähnt, dafs er das Tier einmal Ende der $60 \mathrm{er}$ Jahre in Weidengebüsch an der Pleifse bei Leipzig beobachtet habe. Da aber Fr. offenbar nur besuchsweise hier war, ist ein sicherer Rückschlufs nicht möglich. Wüstner (Sächs. Ber.) hat den Vogel ebenfalls früher bei Leipzig, Pomfsen und Otterwisch gehört. Jacobi und Lohse stellten sein Brüten bei Connewitz, Grofszschocher und Lindenau fest, und R. Müller fand nach mündlicher Mitteilung bei Knauthain in einem Jahre 4 Gelege, von denen ich eins in meiner Sammlung aufbewahre. Hennicke schreibt 1891, dafs er als Sommervogel in grofser Individuenzahl auftrete und 1894, dafs er der häufigste Rohrsänger im Rohrbacher Gebiete sei. Dr. Hesse stellte 1905 dort ca. 30 Brutpaare fest. Für Borna verzeichnen Müller und Ulbricht, dafs die Rohrdrossel am Stadtteiche gebrütet habe und berichten für die Breitingen-Haselbacher Teiche, dafs sie dort nicht seltener, resp. häufiger Brutvogel sei. Nach Schulze (Sächs. Ber.) ist dieser Rohrsänger im Gebiete von Frohburg an allen gröfseren Teichen Brutvogel. Liebe führt ihn als Brutvogel im Elstertale bei Gera an und verzeichnet sein Auftreten bis zum Frankenwald. Chr. L. Brehm berichtet über sein Brüten an den Oberlödlaer Teichen bereits 1834, und Liebe ist der Meinung, dafs das Tier von hier 
und den Haselbacher Teichen aus weiter nach Süd und West vorgedrungen sei. Dr. Koepert führt aufser den Haselbacher und Oberlödlaer Teichen auch die Hainspitzer Teiche als Brutgebiet auf. Im Gebiete der Vereinigten Mulde bezeichnet den Drosselrohrsänger Hülsmann als Sommerbrutvogel für das Wurzner Gebiet. Höpfner registriert ihn für Grimma, resp. für die Nimbscher Teiche und sagt ferner, dafs er an den Altenhainer Teichen der gemeinste Robrsänger sei. Heyder, der ein Männchen auch einmal im Getreidefeld singend beobachtete, gibt an, dafs er überall im Wermsdorfer Teichgebiet, doch nirgends häufig auftrete. Für das Zwickauer Gebiet bezeichnet Berge die Rohrdrossel als Durchzugsvogel. Er erwähnt eines 1896 bei Haara beobachteten Exemplares und zitiert, allerdings ohne Quellenangabe die Beobachtung Wüstners, der 1887 dort 2 Exemplare feststellte. Einen Fall des Vorkommens in der Hohensteiner Gegend konnte ich im Sommer 1915 feststellen. Wilde in Hohenstein legte mir einen ihm unbekannten Vogel im Balge vor, der ihm zum Präparieren aus Obertirschheim übersandt worden war. Offenbar war das Tier, über das ich näbere Angaben nicht erhalten konnte, nur im Zuge erbeutet worden. Meine Ankunftsbeobachtungen für Möckern lauten 6. V. 1894,14 . V. 1895 (ein ziemlich später Termin!) Ankunftstermine sind nach Päfsler 2. V., resp. Ende April und nach Höpfner 2. V. (Gesang). Dr. Hesse notiert für Gundorf 1905 3. V.-23. VIII., 1906 30. IV.-18. VIII., für Rohrbach 1905 5. V.-21. VII. und 1906 27. IV.-3. IX., zusammenfassend als Aufenthaltszeit Ende April-Mitte September. Jacobi und Lohse verlegen den Abzug in die letzten Tage des August, und Pälsler gibt die Abzugszeit je einmal für Mitte September und Ende August an. Die Nester stehen in der Regel über dem Wasser im Rohre, doeh traf den Vogel R. Müller (Ornith. Monatsschr. 1891) auch an einem trockenen Flufsufer brütend. 1894 fand derselbe Beobachter ein Nest in einem mit Rohr durchsetzten Schwarzdornbusch am Pleifsenufer, was ich 2 Jahre später an derselben Stelle erneut konstatieren konnte. Die volle Zahl der Eier des Geleges beträgt in recht konservativer Weise 5 Stück und dürfte infolge des um die Mitte des Maies noch nicht genügend geschlossenen Rohres kaum vor dem letzten Drittel des Monats begonnen werden. R. Müller fand 5 er Gelege am 6. VI. und 30. VI. Am 27. V., 10. VI. und 25. VI. fand ich die Nester mit 5 frischen, 5 einige Tage bebrüteten Eiern und 5 Jungen. Hagelweid sammelte ein Gelege mit frischen Eiern am 2. VI. Dr. Hesse beobachtete am 2. VI. den Nestbau, der am 13. VI. 5 frische Eier enthielt. Bei der Notiz über flügge Junge schon am 1. VI. ist offenbar ein Druckfehler übersehen worden. Päfsler registriert Eier für den 20. VI. und 12. VII. und Heyder für den 2. VII. Sechser- und Vierergelege, wie ich sie in meiner Sammlung von Walternienburg und aus der Havelgegend besitze, sind mir aus hiesiger Gegend nicht bekannt geworden. Dr. Proft aber teilt 
mir mit, dafs in normalen Jahren im Haselbacher Teichgebiet die Sechszahl im Gelege die Regel sei.

\section{Acrocephalus schoenobaenus (L.).}

Nach dem vorliegenden eigenen und fremden Beobachtungsmaterial steht der Schilfrohrsänger im Leipziger Augebiete hinsichtlich seiner Häufigkeit und seiner Verbreitung dem Sumpf-, Teich- und Drosselrohrsänger entschieden nach. Am häufigsten noch traf ich den Vogel während der Zug- und Brutzeit im Möckernschen Ausschachtungsgebiet, zur Zugzeit öfters recht häufig, z. B. am 3. V. 1893, am 20. V. 1896 und auch im Jahre 1913 wieder 2 Exemplare. Vermutlich noch im Durchzuge beobachtete ich ihn auch in Crostewitz am 13. V. 1896. 1904 beobachtete Dr. Hesse diesen Rohrsänger in Gundorf auf dem Durchzuge, stellt aber schon ein Jahr später für diese Örtlichkeit 10 Brutpaare fest. Die weitere Angabe des zuverlässigen Autors, dafs der Schilfrohrsänger für das Leipziger Gebiet als häufiger Brutvogel zu bezeichnen sei, möchte ich nach meinen Feststellungen dahin abändern, dafs diese Verallgemeinerung nur für recht wenige Stellen zutreffen dürfte. Jacobi und Lohse beobachteten den Schilfrohrsänger auf dem Herbstzuge. Prof. Voigt berichtet über sein Vorkommen in den dichten Weidengebüschen an den Ufern der Flüsse und in den Ausstichsümpfen bei Grofszschocher und Möckern. Auch Lindner verzeichnet ihn für letzteren Ort, und Dr. Proft besitzt ein Sechsergelege aus demselben Gelände. Hennicke selbst fand den Vogel nicht im Rohrbacher Gebiet, soll aber nach seinen zuverlässigen Gewährsmännern dort brüten, was durch Dr. Hesse bestätigt wird, der dort 190410 Brutpaare feststellt. Nach Liebe kommt er im ganzen Gebiet vor, aber allenthalben nicht häufig. Fundorte werden leider nicht angeführt. PäIsler berichtet: „Bei Haselbach“. Dr. Proft aber hat ihn dort noch nicht feststellen können, wo ibn nach Dr. Koepert Schlegel schon 1822 als brütend verzeichnet. Im übrigen bezeichnet ihn Koepert, wie Liebe, als seltenen Brutvogel. Er soll früher viel häufiger gewesen sein, da Kratzsch den Vogel als gemein an den Lödlaer Teichen bezeichnet hat. Einer kurzen Angabe Bräss' entnehme ich, dafs er auch im Frohburger Gebiet auftritt. Berge verzeichnet ihn als vereinzelten Brutvogel an sumpfigen Stellen mit Weidengebüsch. Bedauerlich bleibt, dafs er die Orte seines Vorkommens überhaupt nicht nennt. Ebenso begründet er die Bemerkung nicht, dafs er früher häufiger gewesen sein soll. Im 5. Jahresberichte für Sachsen gibt Schurig an, dafs er bei Planitz bei Zwickau während des Frübjahrszuges am 25. IV. 1 Exemplar am Bachufer beobachtet habe. Ich kenne diesen Beobachter als Ornithologen zwar nicht, da aber die Zugzeit stimmt, ist die Beobachtung vielleicht nicht ganz von der Hand zu weisen, obwohl sie auch auf jeden andern Rohrsänger passen dürfte. Für 
das Gebiet der Vereinigten Mulde berichtet Höpfner für Grimma von einem singenden Exemplar am 7. VII. und in späteren Jahren von 2 Exemplaren auf dem Frühjahrszuge an den Teichen der Promenade. Nach seinem Dafürhalten (Ornith. Monatsschr. 1906) scheint der Schilfrohrsänger im Gebiete also nur als Durchzugsvogel aufzutreten. Im Gebiete der Wermsdorfer Teiche begegnete ihm Heyder öfters, resp. vereinzelt. Er scheint nach ihm dort sparsamer Brutvogel zu sein. Prof. Voigt registriert seine Ankunft für Ende April. Am 24. IV. 1895 stellte Thienemann (nach mündl. Mitteil.) seine Ankunft in Möckern fest. Am 6. V. 1896 aber erst traf ich den Vogel erstmalig im dortigen Gebiet an. Dr. Hesse gibt für 1905-06 folgende Aufenthaltsdauer an: Gundorf : 15. IV.-26. VIII., 18. IV.-15. IX., Rohrbach: 13. IV.18. VIII., 20. IV.-17. IX. Auffällig bleibt die etwas grofse Differenz in der Abzugszeit zweier aufeinanderfolgender Jahre. Höpfner stellte den Durchzug von 2 Exemplaren am 6. V. fest. Jacobi und Lohse beobachteten ihn auf dem Herbstzuge unter anderen „Schilfsängern" am 10. IX. Heyder beobachtete den Herbstzug von wenigen Exemplaren bis zum 18. IX. Betreffs der Nistzeit erwähne ich, dafs Thienemann auf gemeinsamen Exkursionen mit mir am 31. V. und 19. VII. 1894 die Nester mit 3 frischen Eiern, resp. 4 halberwachsenen Jungen und 1 unbefruchteten $\mathrm{Ei}$ auffand. Letzteres bewahre ich als Belegstück nach auf. Die Nester standen auf dem Trockenen in der Nähe der Lachen, wenig hoch über dem Boden in Rohr- oder Weidenstubben, was auch Dr. Proft bestätigt. Am 20. VI. 1895 beobachtete ich das Füttern der ausgeflogenen Jungen und fing am 22. VI. ein Exemplar als Belegstück für meine Sammlung.

\section{Acrocephalus aquatica (Gm.).}

Eine sichere Nachricht über das Vorkommen des Binsenrohrsängers im Leipziger Gebiet erhielten wir durch Jacobi und Lohse, nach welchen am 1. VIII. 1889 ein Exemplar im Bruche bei Grofszschocher beobachtet wurde. Ich erinnere mich noch lebhaft, wie Prof. Jacobi mir gegenüber auch persönlich wiederholt dieses Vorkommnisses mit Freude und Stolz gedachte. Weitere einwandfreie Nachrichten über das Auftreten des Vogels erhalten wir durch Dr. Hesse, der ihn während dreier Spätsommer resp. Herbste für Gundorf auf dem Durchzuge nachwies. R. Müller will den Binsenrohrsänger auch einmal bei Knauthain während der Brutzeit beobachtet haben. Ich führe diese Mitteilung der Vollständigkeit halber mit an. Nach Prof. Hennicke soll der Vogel noch zuverlässigen Gewährsmännern im Gebiete der Rohrbacher Teiche auch brüten. Da dieses Gebiet von tüchtigen hiesigen Ornithologen auch bis in die neueste Zeit gründlich studiert wurde, der Vogel aber von keinem nachgewiesen wird, ist der Notiz keine allzuhohe Bedeutung beizumessen, zumal Prof. 
Hennicke nicht persönlich für die Richtigkeit einstehen kann. Dem scharfsinnigen und beobachtungsvielseitigen Dr. Hesse, der das Tier dort nur einmal im Zuge beobachtete, wäre ein Brutvorkommnis sicher nicht entgangen. Nach Dr. Koepert kommt er auch südlich von Leipzig, im Altenburger Gebiet, brütend nicht vor, wurde aber auf dem Durchzuge beobachtet, z. B. von Kratzsch im Herbst 1858 im Gebiet der Frohburg-Eschefelder Teiche. Das Vorkommen des Binsenrohrsängers im Leipziger Gebiet bleibt somit ein sehr seltenes, auf den Durchzug beschränktes, aber nach Dr. Hesse vielleicht regelmäfsiges. Nach letzterem Autor dürfte er in der Zeit von Ende Juli bis Anfang Oktober zu suchen sein.

\section{Locustella naevia naevia (Bodd.).}

Der Heuschreckenrohrsänger ist im Leipziger Gebiete keiue häufige und alltägliche Erscheinung und sein Auftreten ein ziemlich zerstreutes. Im Gegensatz zu Dr. Hesse möchte ich ihn einen unregelmäfsigen und sehr seltenen Brutvogel aber nicht nennen. Das 1. Mal hörte ich den Vogel in einem kleinen, dichten Weidenheger am Bisthum, einem Walde bei Zwenkau am 5. V. 1895, schwirren, am 17. V. 1896 dort wiederum. Ich machte auf das Vorkommnis den mich begleitenden Forstaufseher Zschoppe aufmerksam mit der Bitte, ferner auf das Vorkommen an anderen Stellen des Aubezirks zu achten. Beim nächsten Zusammentreffen berichtete er mir, dafs er den Vogel auch am Eichholze habe schwirren hören. Am 14. VI. und 22. VI. 1895 stellte ich den Vogel auch in 3 Exemplaren in Möckern fest und R. Müller in demselben Jahre auch in einem Kleefelde bei Techwitz. Am 23. V. 1896 beobachtete ich den Schwirrer bei Markkleeberg und Crostewitz, wo R. Müller 3 Exemplare beobachten konnte. Hier fand R. Müller Mitte Juni auch ein frisches Gelege. Dr. Hesse begegnete den Vogel im nordwestlichen Auwaldgebiete, und Prof. Voigt stellte ihn auf dem Durchzuge im Connewitzer Holz, bei Möckern-Wahren und bei Schkeuditz fest. Für das südlich von Leipzig liegende Altenburger Gebiet berichtet Pälsler, dafs er den Vogel in den 40 er Jahren das erstemal bei Altenburg beobachtet habe, dafs er sich mit den Jahren sehr vermehrte, dafs er bis 1880 häufig in Kornfeldern vorkam, nach und nach abgenommen habe und nach 2 Jahren ganz verschwunden sei. Ein 1889 wieder aufgetretenes Paar wurde durch Hagelwetter erschlagen. Im 87 er Sächs. Ber. erwähnt dieser gute Kenner einheimischer Vögel, dals der Vogel in der Altenburger Gegend jetzt selten sei. Prof. Liebe erwähnt nach Kratzsch sein Vorkommen zwischen Śchmölln und Kleintauschwitz, wo man stellenweise auf einer halben Stunde Wegs durchschnittlich 3 Pärchen in Roggen-, Gersten- oder Kleefeldern antreffen könne. Dr. Koepert wiederholt im wesentlichen dasselbe und bomerkt, dafs der Feldschwirl 
nach Heller bei Schmölln und im Pleifsentale nicht selten sein soll. Als weitere Orte des Vorkommens werden ferner angeführt: Kauritz, Lähmigen, Hainichen, Ponitz, Merlach, Bernshain, Möckern, Grolsstäbnitz. Auch fürs Gebiet von Meusdorf-Kohren verzeichnet ihn Schulze als Brutvogel in jungen Kulturen, die an Felder angrenzen und in Roggen- und Rapsfeldern. Noch südlicher beobachtete ihn Päfsler bei Breitenbach-Meerane auf dem Durchzuge. Aus dem Gebiete der Vereinigten Mulde fehlen Nachrichten über sein Auftreten gänzlich. Nur Berge bezeichnet den Rohrschwirl im Gebiete der Zwickauer Mulde als seltenen Durchzugsvogel auf Grund eines am 18. V. 1894 bei Zwickau schwirrenden Exemplars. Von Zugnotizen gibt Päfsler den 8. V. an, Schulze das Eintreffen am 10. V. Lindner hörte das Schwirren einmal am 14. V., und Dr. Hesse notiert für die Zeit seines Verweilens Mitte Mai bis Mitte September. Wenn ich Päfslers Abkürzung recht deute, notiert er einmal Eier für den 26. VI.

\section{Locustella fluviatilis (Wolf).}

Hinsichtlich des Flufsrohrschwirls schreibt Lindner in seiner Arbeit 1897: „In dem so günstigen Terrain der Auwälder und Wiesen bei Leipzig habe ich den Vogel nie angetroffen". Lindners Beobachtungen beziehen sich auf die Zeit von Anfang bis Mitte der 80 er Jahre. Am 14. V. 1895 verhörte ich im Möckernschen Gebiete in einem Weidenbusche an einer jetzt ausgefüllten Lache am Bahnübergange einen vermeintlichen Heuschreckenrohrsänger und notierte in mein Tagebuch: „L. naevia gehört, schwirrte mehr in errrr, ähnlich dem Schlufston im Grauammergesang - $\boldsymbol{L}$. fluviatilis? ??". Da ich den Gesang von L. fluviatilis nicht kannte, wagte ich kein bestimmtes Urteil, sondern hielt den Gesang für eine individuelle Gesangsleistung des Heuschreckenrohrsängers. $\mathrm{Da}$ das Vorkommen des Schlagschwirls für das Leipziger Gebiet nun in späteren Jahren einwandfrei nachgewiesen wurde, behaupte ich vielleicht nicht zu viel, wenn ich annehme, dafs es sich damals bereits, wenn auch von mir unerkannt, vielleicht um einen solchen gehandelt hat. Dr. Hesse gebührt das Verdienst, den Schlagschwirl für das hiesige Gebiet in den Jahren 1907/09 im nordwestlichen Auwaldgebiete einwandfrei nachgewiesen zu baben. Ich kann mich weiterer Mitteilungen hierüber enthalten und verweise auf die interessanten Mitteilungen des verdienstvollen Autors im J. f. 0.1909 pag. 26 und 359 und 1910 pag. 516. Am 2. VII. 1912 teilte mir Koll. Nestler, ein ausgezeichneter Beobachter und Kenner mit, dafs er den Vogel im gleichen Jahre bei Möckern wiederholt habe schwirren hören und am 28. VI, auch das Füttern der Jungen beobachtet habe. Somit wurde der Schlagschwirl als ein zwar seltener, aber doch hin und wieder als ein hier brütender Vogel zu bezeichnen sein. Ein ganz unanfechtbares und sicheres Urteil ist nach dem Stande unserer gegenwärtigen Kenntnis also nicht 
so leicht zu fällen. Schulze bezeichnet den Schlagschwirl für das Gebiet Frohburg-Kohren als Durchzugsvogel. Ich würde dieser Mitteilung keinerlei Bedeutung beigemessen haben, wenn aus den Notizen des Beobachters über den Heuschreckenrohrsänger nicht mit Sicherheit hervorginge, dafs er den Vogel gut kennt. Freilich ist bei dem Fehlen aller Details in einer so wichtigen Sache mit einer so als selbstverständlich und oberflächlich hingeworfenen Bemerkung nicht viel anzufangen. Immerhin ist es aber nicht ganz von der Hand zu weisen, dafs der Vogel zur Beobachtung gekommen sein mag. Von hervorragendem Interesse ist es, dafs Prof. Liebe den Schlagschwirl im Gebiete der oberen Elster, an einem Nebenflusse derselben, an der Gölzsch, in den Jahren 1875 und 1876 nachgewiesen hat. 1877 freilich war der Vogel nicht wieder aufzufinden. Merkwürdigerweise ist von allen Rohrsängerarten kein so südliches Vorkommen und Auftreten gebirgswärts festgestellt worden, als es gerade bei dem seltensten unserer Rohrsänger der Fall ist.

\section{Ornithologische Mittellungen.}

\section{Von Werner Hagen.}

Im Nachstehenden möchte ich einige Beobachtungen, die mir faunistisch oder biologisch wertvoll erscheinen, zur allgemeinen Kenntnis bringen. Die Beobachtungen sind in der Zeit vom 1. August 1915 bis zum 1. August 1916 in der Gegend von Lübeck gemacht.

1. Alca torda L. Am 19. Februar beobachtete ich ein tauchendes Stück auf dem Bretling. So weit flufsaufwärts geht er selten.

2. Colymbus cristatus L. Der Haubentaucher, sonst ein stark abnehmender Brutvogel des lübeckischen Gebietes, hat 1916 sehr zugenommen. Am 19. April sah ich auf einem kleinen Teil der Wakenitz allein 12 Paare. Was aber bedeutet das, dafs bis Ende Mai auf dem Bretling (Untertrave) etwa 70 Stck. sich auf diesem für Brüten ungeeigneten Gewässer aufhielten? Die Vögel waren im vollen Hochzeitsschmuck. Zwar schrieb ich in meinem Werk über die Vögel Lübecks, dafs „im Frühling und Herbst durchziehende H. besonders auf Stau und Bretling liegen", aber zur Brutzeit habe ich hier keine beobachtet. Ob der Krieg schuld ist? Vielleicht sind auch anderorts solche nichtbrütenden Schwärme beobachtet? - Auf den bei Mölln liegenden Lauenburger Seen stellte ich am 27. und 28. April folgende Zahlen fest: Ziegelsee ca. 20 Paare, Schmalsee 2 Paare, Lüttauersee 2 Paare, Drüsensee 20 Paare, Schwarzsee 1 Ex., Pinnsee - Paar. - Sehr zahlreich ist er auf dem Hemmelsdorfer See. 


\section{$2 \mathrm{BHL}$ Biodiversity Heritage Library}

1917. "Die Rohrsänger des Leipziger Flachlandsgebietes mit besonderer Berücksichtigung ihres Vorkommens in den Flufsgebieten der weifsen Elster, Pleifse, vereinigten und Zwickauer Mulde nach dem sächsischen Berglande und Erzgebirge zu." Journal $f u$

r Ornithologie 65, 169-181. https://doi.org/10.1007/bf02250393.

View This Item Online: https://www.biodiversitylibrary.org/item/43317

DOI: https://doi.org/10.1007/bf02250393

Permalink: https://www.biodiversitylibrary.org/partpdf/141718

\section{Holding Institution}

MBLWHOI Library

\section{Sponsored by}

MBLWHOI Library

\section{Copyright \& Reuse}

Copyright Status: No known copyright restrictions as determined by scanning institution.

This document was created from content at the Biodiversity Heritage Library, the world's largest open access digital library for biodiversity literature and archives. Visit BHL at https://www.biodiversitylibrary.org. 